\title{
Atividade antifúngica de fitoterápicos sobre espécies de Candida: uma revisão de literatura
}

\author{
Antifungal activity of herbal medicines on Candida species: \\ a literature review
}

\author{
La actividad antifúngica de las hierbas medicinales \\ en las especies de Candida: una revisión de la literatura
}

\author{
Julliana Cariry Palhano FREIRE ${ }^{\mathbf{1}}$ \\ Marina Tavares Costa NÓBREGA ${ }^{2}$ \\ José Klidenberg de OLIVEIRA-JÚNIOR ${ }^{3}$ \\ Stéphanie Cariry Palhano FREIRE ${ }^{4}$ \\ Eduardo DIAS-RIBEIRO 5 \\ Edeltrudes de Oliveira LIMA ${ }^{6}$

\begin{abstract}
${ }^{I}$ Cirurgiã-Dentista, Mestranda em Ciências Odontológicas, Universidade Federal da Paraíba - UFPB, João Pessoa - PB, Brasil ${ }^{2}$ Cirurgiã-Dentista, Mestranda em Ciências Odontológicas, Universidade Federal da Paraíba - UFPB, João Pessoa - PB, Brasil ${ }^{3}$ Cirurgião-Dentista, Mestrando em Ciências Odontológicas. Universidade Federal da Paraíba - UFPB, João Pessoa - PB, Brasil ${ }^{4}$ Enfermeira. Hospital Universitário Alcides Carneiro, Campina Grande- PB, Brasil ${ }^{5}$ Doutor. Universidade Federal de Campina Grande, Patos- PB, Brasil
\end{abstract} \\ ${ }^{6}$ Doutora. Programa de Pós-Graduação em Odontologia, Universidade Federal da Paraíba - UFPB, João Pessoa - PB, Brasil
}

\begin{abstract}
Resumo
Introdução: A candidíase ou candidíase oral é a infecção fúngica oportunista mais comum, sendo a Candida albicans a espécie mais causadora de infecção no ser humano, principalmente em pacientes imunologicamente comprometidos. Objetivo: $O$ presente estudo teve como objetivo realizar um levantamento da literatura sobre a atividade antifúngica dos produtos naturais a base de plantas contra espécies de Candida. Material e Método: Foi realizado um levantamento da literatura através das bases de dados PUBMED e SCIELO, no período de 2000 a 2016. Resultados: Nove artigos científicos sobre o tema foram encontrados e fizeram parte do estudo. Seis plantas medicinais foram citadas, dentre as quais todas apresentaram atividade antifúngica frente à Candida. Conclusão: Observou-se que as plantas medicinais estudadas apresentaram significativa atividade antifúngica frente a espécies dessa levedura.
\end{abstract}

Descritores: Fitoterapia; Candida albicans; Candida.

\begin{abstract}
Introduction: Candidiasis or oral candidiasis is the most common opportunistic fungal infection, and Candida albicans is the most common cause of infection in humans, especially in immunologically compromised patients. Objective: This study aimed to perform a literature review on the antifungal activity of natural herbal products against Candida species. Material and Methods: A literature review was carried out through the PUBMED and SCIELO databases, from 2000 to 2016. Results: Nine scientific articles on the subject were found and were part of the study. Six medicinal plants were cited, among which all presented antifungal activity against Candida. Conclusion: It was observed that the medicinal plants studied showed significant antifungal activity against yeast species.

Descriptors: Phytotherapy; Candida albicans; Candida.
\end{abstract}

\section{Resumen}

Introducción: La candidiasis o candidiasis oral es la infección fúngica oportunista más común es Candida albicans más especies que causan infecciones en los seres humanos, en particular para los pacientes inmunodeprimidos. Objetivo: Este estudio tuvo como objetivo realizar un estudio de la literatura sobre la actividad antifúngica de productos naturales a base de plantas contra especies de Candida. Material y Métodos: Una revisión de la literatura se llevó a cabo a través de las bases de datos PubMed y SciELO, de 2000 a 2016. Resultados: Nueve artículos científicos sobre el tema se encontraron y fueron parte del estudio. Se mencionaron Seis plantas medicinales, entre las que todos mostraron actividad antifúngica frente a Candida. Conclusión: Se observó que las plantas medicinales estudiadas mostraron actividad antifúngica significativa contra las especies de esta levadura.

Descriptores: Fitoterapia; Candida albicans; Candida.

\section{INTRODUÇÃO}

Candidíase ou candidose é uma micose causada por leveduras do gênero Candida, em que a lesão pode ser branda, aguda ou crônica, superficial ou profunda, e de espectro clínico bem variável. O principal agente das candidíases é a $C$. albicans, constituindo na maioria dos estudos $60 \%$ dos isolados de amostras clínicas, além de outras espécies como C. parapsilosis, C. tropicalis, C. krusei, C. glabrata e $C$. guillermondii, que também contribuem para o desenvolvimento desta doença ${ }^{1}$. C. albicans é, sem dúvida, a espécie mais frequentemente isolada de infecções 
superficiais e invasivas em diversos sítios anatômicos e como causa de candidíase em todas as partes do mundo. É a espécie de Candida com maior conhecimento patogênico, devido à diversidade de fatores de virulência descobertos. Habitualmente se considera que a origem de $C$. albicans causadora de infecções seja a microbiota do trato digestório humano (organismo comensal). C. albicans foi o primeiro fungo zoopatogênico que teve o seu genoma sequenciado (organismo diploide com oito pares de cromossomos), o que possibilita uma variedade de experimentos $\mathrm{e}$, por conseguinte, um grande avanço na biologia deste fungo, principalmente na expressão dos genes ${ }^{2}$.

Espécies de Candida residem como comensais, fazendo parte da microbiota normal dos indivíduos saudáveis. Todavia, quando há uma ruptura no balanço normal da microbiota ou o sistema imune do hospedeiro encontra- se comprometido, as espécies do gênero Candida tendem a manifestações agressivas, tornando-se patogênicas ${ }^{3}$.

As manifestações clínicas causadas pela Candida são variadas, podendo gerar uma infecção localizada de mucosas até uma doença disseminada potencialmente fatal. $\mathrm{O}$ principal fator que determina o tipo e extensão de infecção causada pela Candida é a resposta imunológica do paciente. A candidíase é a infecção oportunista mais comumente relatada em pacientes com AIDS $^{4}$.

Numerosos fatores contribuem para as infecções fúngicas, dentre os quais podemos destacar: o rompimento das barreiras cutânea e mucosa, disfunção dos neutrófilos, defeito na imunidade mediada por células, desordem metabólica, exposição direta aos fungos, extremos de idade (recém-nascidos e idosos), desnutrição aguda, longo tratamento com antibióticos, quimioterapia, transplantes, resistência a antifúngicos, dentre outros 5 .

Tem-se observado principalmente no ambiente hospitalar, o aumento de infecções por Candida spp resistentes a antifúngicos. Esta resistência tem elevado a taxa de insucesso na terapêutica contra essa infecção, assim como o crescimento da morbidade e da mortalidade ${ }^{6}$.

A C. albicans é naturalmente sensível a todas as drogas antifúngicas de uso sistêmico, mas casos de resistência adquirida a azólicos são conhecidos em pacientes expostos prolongadamente a estes medicamentos. Quanto à resistência a anfotericina $\mathrm{B}$, os relatos são mínimos ${ }^{2}$.

$\mathrm{O}$ uso excessivo destes fármacos propicia o surgimento de leveduras resistentes, principalmente em pacientes imunossuprimidos, susceptíveis a infecções frequentes. Assim, há necessidade do desenvolvimento de novos fármacos de maior eficácia, dentre as possibilidades, surge a utilização de fitoterápicos como tratamento alternativo. Os mesmos se diferem por apresentar uma diversidade molecular superior aos sintéticos, proporcionando novas descobertas, com pesquisa nas atividades biológicas que podem favorecer na prevenção e tratamento de doenças ${ }^{7}$.

Moléculas isoladas de produtos naturais com potencial antifúngico podem contribuir para a indústria farmacêutica no desenvolvimento de fármacos e para a produção de novas formulações, com menos efeitos colaterais, amplo espectro de ação e menor custo ${ }^{8}$. A atividade antimicrobiana de extratos vegetais é avaliada através da determinação de uma pequena quantidade da substância necessária para inibir o crescimento do microrganismo-teste; esse valor é conhecido como Concentração Inibitória Mínima (CIM) ${ }^{9}$.

O território brasileiro possui cerca de $20 \%$ da biodiversidade mundial, incluindo plantas, que servem como matéria-prima para a produção de medicamentos fitoterápicos e outros. A grande diversidade cultural e étnica do Brasil é responsável pelo conhecimento transmitido ao longo de gerações sobre a gestão e uso de plantas medicinais ${ }^{10}$.

Tendo em vista a alta frequência de infecções pelas espécies de Candida, assim como o aumento das pesquisas envolvendo o uso de fitoterápicos para combater essa levedura, o presente estudo teve como objetivo realizar um levantamento da literatura sobre a atividade antifúngica dos produtos naturais a base de plantas contra cepas de Candida, no período de 2000 a 2016.

\section{MATERIAL E MÉTODO}

Esse estudo consistiu de uma revisão de literatura baseada na pesquisa de artigos científicos obtidos das bases de dados PUBMED (http://www.ncbi.nlm.nih.gov/pubmed) e SCIELO (http://www.scielo.org), no período compreendido entre 2000 a 2016. Foram utilizadas as seguintes palavraschaves: (1) Português - fitoterápico, Candida albicans, antifúngico; Candida, como também as respectivas palavras no plural (2) Inglês - phytoterapy, Candida albicans, antifungal, Candida. Os critérios de inclusão aceitos foram: o ano do estudo, sendo considerados os anos de 2000 a 2016 , foram inseridos também os artigos que se adequaram ao tema proposto, utilizando plantas medicinais para a análise de sua atividade antifúngica frente às espécies de Candida, que se trataram de ensaios clínicos randomizados e estudos observacionais. Os artigos científicos que não se enquadraram aos critérios de inclusão foram excluídos da pesquisa.

\section{RESULTADOS E DISCUSSÃO}

Pelo levantamento bibliográfico, foram encontrados e incluídos no estudo nove artigos científicos que abordaram o tema em questão, estavam dentro do período considerado e se tratavam de ensaios clínicos randomizados ou estudos observacionais. Seis plantas medicinais foram citadas, nas quais todas demonstraram atividade antifúngica frente à Candida (Tabela 1).

As plantas mais estudadas foram: Melaleuca atnerfolia (árvore-de-chá), Allium sativum (alho) e Ricinus communis (mamona). A atividade antifúngica observada pela família Punicaceae no trabalho pesquisado de Vasconcelos ${ }^{11}$ et al., (2003), pode estar relacionada à presença de glicosídeo flavanóide e taninos, componentes que apresentam propriedades antimicrobianas e anti-inflamatórias ${ }^{12}$. Há um interesse crescente no uso de taninos como agentes antimicrobianos. A atividade de taninos contra bactérias e leveduras pode ser medida por sua ação sobre as membranas, já que estes podem atravessar a parede celular, composto por vários polissacáridos e proteínas, e se ligarem à sua superfície ${ }^{13}$.

Estudos com produtos naturais geram dificuldades no que concerne à comparação dos resultados, tal situação devese às diferentes apresentações dos produtos utilizados (tintura, extrato etanólico, óleo essencial, dentre outros), bem como aos variados critérios metodológicos empregados ${ }^{14}$. Os estudos demonstraram formas de apresentação diferentes do fitoterápico, entre elas, soluções orais, gel e chá por decocção. Dentre os tipos de estudo, a maioria dos artigos se trataram de ensaios clínicos randomizados.

A maior ou menor atividade biológica dos óleos essenciais tem se mostrado dependente da composição de seus constituintes químicos como citral, pineno, cineol, cariofileno, elemeno, furanodieno, imoneno, eugenol, 
eucaliptol, carvacrol e outros. Estes constituintes são responsáveis pelas propriedades antissépticas, antibacterianas, antifúngicas e antiparasíticas ${ }^{15}$. O modo de extração dos princípios ativos pode influenciar significantemente na atividade antimicrobiana. A biossíntese dos constituintes de uma planta é fortemente afetada pelo ambiente, colheita e pós-colheita, precipitação pluviométrica, temperatura, luminosidade e umidade ${ }^{16}$.

Tabela 1. Estudos sobre atividade antifúngica de fitoterápicos contra espécies de Candida*

\begin{tabular}{|c|c|c|c|c|}
\hline Autor (ano) & $\begin{array}{l}\text { Planta } \\
\text { medicinal } \\
\text { (espécie) }\end{array}$ & $\begin{array}{c}\text { Nome } \\
\text { Popular }\end{array}$ & Controle & $\begin{array}{c}\text { Sensibilidade } \\
\text { da Candida } \\
\text { frente ao } \\
\text { fitoterápico }\end{array}$ \\
\hline $\begin{array}{l}\text { Vazquez e } \\
\text { Zawawi } \\
(2002)^{21}\end{array}$ & $\begin{array}{l}\text { Melaleuca } \\
\text { atnerfolia }\end{array}$ & $\begin{array}{l}\text { Árvore- } \\
\text { de-chá }\end{array}$ & Fluconazol & + \\
\hline $\begin{array}{l}\text { Vasconcelos } \\
\text { et al. }(2003)^{11}\end{array}$ & $\begin{array}{c}\text { Punica } \\
\text { granatum }\end{array}$ & Romã & Miconazol & + \\
\hline $\begin{array}{l}\text { Sabitha et al. } \\
\quad(2005)^{22}\end{array}$ & $\begin{array}{l}\text { Allium } \\
\text { sativum }\end{array}$ & Alho & $\begin{array}{c}\text { Clotrimazo } \\
1\end{array}$ & + \\
\hline $\begin{array}{l}\text { Catalán et al. } \\
(2008)^{23}\end{array}$ & $\begin{array}{l}\text { Melaleuca } \\
\text { atnerfolia }\end{array}$ & $\begin{array}{l}\text { Árvore- } \\
\text { de-chá }\end{array}$ & Nistatina & + \\
\hline $\begin{array}{l}\text { Paiva et al. } \\
(2009)^{24}\end{array}$ & $\begin{array}{c}\text { Uncaria } \\
\text { tomentosa }\end{array}$ & $\begin{array}{l}\text { Unha de } \\
\text { gato }\end{array}$ & Miconazol & + \\
\hline $\begin{array}{l}\text { Wright et al. } \\
(2009)^{25}\end{array}$ & $\begin{array}{l}\text { Cymbopogon } \\
\text { citratus }\end{array}$ & $\begin{array}{l}\text { Capim- } \\
\text { limão }\end{array}$ & $\begin{array}{c}\text { Violeta de } \\
\text { Genciana } \\
0,5 \%\end{array}$ & + \\
\hline $\begin{array}{l}\text { Bakhshi et al. } \\
\qquad(2012)^{26}\end{array}$ & $\begin{array}{l}\text { Allium } \\
\text { sativum }\end{array}$ & Alho & Nistatina & + \\
\hline $\begin{array}{l}\text { Pinelli et al. } \\
\quad(2013)^{27}\end{array}$ & $\begin{array}{l}\text { Ricinus } \\
\text { communis }\end{array}$ & Mamona & $\begin{array}{l}\text { Nistatina e } \\
\text { Miconazol }\end{array}$ & + \\
\hline $\begin{array}{l}\text { Salles et al. } \\
(2015)^{28}\end{array}$ & $\begin{array}{l}\text { Ricinus } \\
\text { communis }\end{array}$ & Mamona & $\begin{array}{c}\text { Solução } \\
\text { salina } \\
0,85 \%\end{array}$ & + \\
\hline
\end{tabular}

A candidíase oral não é uma enfermidade mortal, mesmo que, ao provocar moléstias de diferentes níveis, compromete o paladar e a deglutição, levando a uma diminuição do apetite, principalmente nos casos de pacientes HIV-positivo, pacientes hospitalizados e idosos. A candidíase oral é a porta de entrada para complicações da candidíase do tipo orofaríngeas, esofágicas, laringianas e sistêmicas ${ }^{17}$.

Candidíase é uma micose de importância em saúde pública, incluída também como doença sexualmente transmissível (DST). São diversas as espécies já reconhecidas como agentes causais, embora a mais bem estudada seja a $C$. albicans, já que o seu isolamento e sua identificação são mais confirmados. As diferentes espécies, com características sutis ou maiores que as diferenciam, apresentam manifestações clínicas e micromorfológicas similares, com flexibilidade para adaptar-se em diferentes sítios anatômicos que, dependendo de condições predisponentes do hospedeiro, podem causar ampla gama de danos ao paciente ${ }^{18}$.

O tratamento da candidíase oral é simples nos pacientes imunocomprometidos ou com imunodepressão leve, em que geralmente os antifúngicos tópicos apresentam resultados eficazes. No entanto, nos casos de imunodepressão o problema maior está na alta taxa de recorrências ou recidivas, requerendo a combinação de uma terapia intensiva tanto sistêmica como local. Em alguns casos se inclui propor a possibilidade de instaurar um tratamento profilático com derivados azólicos, como nos pacientes com HIV. Apesar dos excelentes resultados com antifúngicos azólicos orais, encontramos formas clínicas de candidíases orais crônicas rebeldes ao tratamento. A retirada dos fatores predisponentes, combinada com derivados azólicos ou poliênicos (nistatina), é o principal tratamento ${ }^{17}$.

C. albicans é a principal levedura isolada, principalmente em infecções nosocomiais, e o principal agente de fungemia no mundo. A sensibilidade desse agente a antifúngicos tem sido observada igualmente no mundo e no restante do Brasil ${ }^{19}$. A utilização de plantas medicinais pode constituir uma alternativa terapêutica bastante útil devido a sua eficácia, baixo custo operacional, aliada a relativa facilidade para a aquisição destas pela população e compatibilidade com a cultura regional ${ }^{14}$.

Considerando a resistência das leveduras pertencentes ao gênero Candida frente aos antifúngicos atualmente utilizados, pode-se inferir que a busca de novos compostos antifúngicos de origem vegetal mostra-se de relevante significância. Há a necessidade de realização de estudos de cunho toxicológico e clínico como suporte de segurança para o uso destes produtos como fármacos ${ }^{20}$.

\section{CONCLUSÃO}

Com base na metodologia empregada e nos resultados encontrados concluiu-se que as plantas medicinais pesquisadas apresentaram resultado satisfatório na atividade antifúngica frente às espécies de Candida.

\section{REFERÊNCIAS}

1. Menezes EA, Cavalcante MS, Farias RB, Teixeira AB, Pinheiro FG, Bezerra BP, et al. Frequência e atividade enzimática de Candida albicans isoladas da mucosa bucal de crianças de uma creche da prefeitura de fortaleza. J Bras Patol Med Lab. 2005; 41(1):9-13.

2. Tiraboschi IN, Carnovale S, Benetucci A, Fernández N, Kurlat I, Foccoli M, et al. Brote de candidemia por Candida albicans em neonatología. Rev Iberoam Micol. 2007; 24(4):263-7.

3. Monge RA, Román E, Nombela C, Pla J. The MAP kinases signal transduction network in Candida albicans. Microbiology 2006; 152(Pt 4):905-12.

4. Kauffman CA. Candidíase. In: Goldman L, Ausiello D. Cecil: Tratado de Medicina Interna. 22. ed. Rio de Janeiro: Elsevier. 2005; p.2713-17

5. Pfaller MA, Diekema DJ. Epidemiology of invasive candidiasis: a persistente public health problem. Clin Microbiol Rev. 2007; 20(1):133-63.

6. Vandeputte P, Larcher G, Bergès T, Renier G, Chabasse D, Bouchara JP. Mechanisms of azole resistance in a clinical isolate of Candida tropicalis. Antimicrob Agents Chemother. $2005 ; 49(11): 4608-15$.

7. Andrade MA, Cardoso MG, Batista LR, Mallet ACT, Machado SMF. Óleos essenciais de Cymbopogon nardus, Cinnamomum zeylanicum e Zingiber officinale: composição, atividades antioxidante e antibacteriana. Rev Ciênc Agron. 2012; 43(2):399-408.

8. Mesa Arango AC, Bueno Sánchez JG, Betancur-Galvis LA. Productos naturales con actividad antimicótica. Rev Esp Quimioterap. 2004; 17(4):325-31.

9. Pinto TJA, Kaneko TM, Ohara MT. Controle Biológico de Qualidade de Produtos Farmacêuticos, Correlatos e Cosméticos. 2 ed. São Paulo: Atheneu; 2003.

10. Brasil. Ministério da Saúde. Política Nacional de Práticas Integrativas e Complementares no SUS - PNPIC-SUS. Brasília: Ministério da Saúde, 2006a. 96p. 
11. Vasconcelos LC, Sampaio MCC, Sampaio FC, Higino JS. Use of Punica granatum as an antifungal agent against candidosis associated with denture stomatitis. Mycoses. 2003 Jun;46(5-6):192-6.

12. Pereira JV, Pereira MSV, Higino JS, Sampaio FC, Alves PM, Araújo CRF. Estudos com Extrato da Punica granatum Linn. (Romã): Efeito antimicrobiano in vitro e avaliação clínica de um dentifrício sobre do microorganismos biofilme dental. Revista Odonto Ciência - Fac. Odonto/PUCRS. 2005;20(49).

13. Vasconcelos LCS, Sampaio FC, Sampaio MCC, Pereira MSV, Higino JS, Peixoto MHP. Minimum Inhibitory Concentration of Adherence of Punica granatum Linn (pomegranate) Gel Against S. mutans, S. mitis and C. albicans. Braz Dent J. 2006; 17(3):223-7.

14. Freires IA, Alves LA, Jovito VC, Almeida LFD, Castro RD, Padilha WWN. Atividades antibacteriana e antiaderente in vitro de tinturas de Schinus terebinthinfolius (Aroeira) e Solidago microglossa (Arnica) frente a bactérias formadoras do biofilme Dentário. Odontol Clín-Cient. 2010;9(2):139-43.

15. Souza EL, Lima EO, Freire KRL, Sousa CP. Inhibitory action of some essential oils and phytochemicals on the growth of moulds isolated from foods. Braz Arch Biol Technol, 2005; 48(2):245-50.

16. Sharma N, Tripathi A. Effects of Citrus sinensis (L.) Osbeck epicarp essential oil on growth and morphogenesis of Aspergillus niger (L.) Van Tieghem. Microbiol Res. 2006;163(3):337-44.

17. Aquirre Urizar JM. Candidiasis orales. Rev Iberoam Micol. 2002; 19(1):17-21.

18. Barbedo LS, Sgarbi DBG. Candidíase. J Bras Doenças Sex Transm. 2010; 22(1):22-38

19. Badiee P, Alborzi A. Susceptibility of clinical Candida species isolates to antifungal agents by E-test, Southern Iran: a five year study. Iran J Microbiol. 2011; 3(4):1838.

20. Lima IO, Oliveira RAG, Lima EO, Farias NMP, Souza EL. Atividade antifúngica de óleos essenciais sobre espécies de Candida. Revista Brasileira de Farmacognosia. 2006;16(2):197-201.

21. Vazquez JA, Zawawi AA. Efficacy of alcohol-based and alcohol-free melaleuca oral solution for the treatment of fluconazole-refractory oropharyngeal candidiasis in patients with AIDS. HIV Clin Trials. 2002 SepOct;3(5):379-85.

22. Sabitha P, Adhikari PM, Shenoy SM, Kamath A, John $\mathrm{R}$, Prabhu MV, et al. Efficacy of garlic paste in oral candidiasis. Trop Doct. 2005;35(2):99-100.

23. Catalán A, Pacheco JG, Martínez A, Mondaca MA. In vitro and in vivo activity of Melaleuca alternifolia mixed with tissue conditioner on Candida albicans. Oral Surg Oral Med Oral Pathol Oral Radiol Endod. 2008;105(3):327-32.

24. Paiva LCA, Ribeiro RA, Pereira JV, Oliveira NMC. Avaliação clínica e laboratorial do gel da Uncaria tomentosa (Unha de Gato) sobre candidose oral. Revista Brasileira de Farmacognosia, Curitiba, 19(2): 423-428, Apr-June. 2009.

25. Wright SC, Maree JE, Sibanyoni M. Treatment of oral thrush in HIV/AIDS patients with lemon juice and lemon grass (Cymbopogon citratus) and gentian violet. Phytomedicine. 2009;16(2-3):118-24.

26. Bakhshi M, Taheri JB, Shabestari SB, Tanik A, Pahlevan R. Comparison of therapeutic effect of aqueous extract of garlic and nystatin mouthwash in denture stomatitis. Gerodontology.2012; 29(2):e680-4.

27. Pinelli LA, Montandon AA, Corbi SC, Moraes TA, Fais LM. Ricinus communis treatment of denture stomatitis in institutionalised elderly. J Oral Rehabil. 2013; 40(5):37580.

28. Salles MM, Badaró MM, Arruda CN, Leite VM, Silva $\mathrm{CH}$, Watanabe E, Oliveira V de C, Paranhos H de F. Antimicrobial activity of complete denture cleanser solutions based on sodium hypochlorite and Ricinus communis - a randomized clinical study. J Appl Oral Sci. 2015;23(6):637-42.

\section{CONFLITO DE INTERESSES}

Os autores declaram não haver conflitos de interesse.

\section{AUTOR PARA CORRESPONDÊNCIA}

\section{Julliana Cariry Palhano Freire}

jullianapalhano@hotmail.com

Submetido em 11/11/2016 Aceito em 12/12/2016 